Linguistique, littérature, didactique

151-152 | 2011

Anthropologies de la littérature

\title{
La Princesse de Clèves et le processus de civilisation
}

\section{Anne Löcherbach}

\section{(2) OpenEdition \\ Journals}

Édition électronique

URL : http://journals.openedition.org/pratiques/1802

DOI : 10.4000/pratiques. 1802

ISSN : 2425-2042

\section{Éditeur}

Centre de recherche sur les médiations (CREM)

\section{Édition imprimée}

Date de publication : 15 décembre 2011

Pagination : 101-116

\section{Référence électronique}

Anne Löcherbach, "La Princesse de Clèves et le processus de civilisation », Pratiques [En ligne], 151-152 | 2011, mis en ligne le 13 juin 2014, consulté le 19 avril 2019. URL : http:// journals.openedition.org/pratiques/1802 ; DOI : 10.4000/pratiques.1802 


\title{
La Princesse de Clèves et le processus de civilisation
}

\author{
Anne Löcherbach
}

Université de Stuttgart

\section{Elias et la littérature}

Depuis les années 1970, les travaux de Norbert Elias font partie intégrante du répertoire commun des concepts théoriques des sciences humaines en général, et de l'histoire littéraire en particulier. Cependant on s'est souvent contenté, comme le fait remarquer Reiner Wild, de faire allusion à quelques notions éliasiennes plutôt imprécises ${ }^{(1)}$. C'est avec le même regret que L. Volkmann conclut son article sur Elias en affirmant que - en dépit de la large diffusion de ses théories au cours de ces dernières années - on commence seulement à reconnaître et à explorer les nombreuses voies qui mènent à une application de sa pensée à la littérature ${ }^{(2)}$. La présente analyse d'une œuvre majeure de la littérature française du XVII siècle se propose d'élucider jusqu'à quel point les études d'Elias rendent plus intelligible un roman qui ne cesse de soulever, depuis sa parution, les questions de ses lecteurs, et de la critique ${ }^{(3)}$.

(1) R. Wild, «Literatur und Zivilisationstheorie », Literaturwissenschaft-Kulturwissenschaft. Positionen, Themen, Perspektiven, éd. par Renate Glaser et Matthias Luserke, Opladen, 1996, pp. 69 et 76.

(2) L. Volkmann, «Elias, Norbert», Metzler-Lexikon Literatur-und Kulturtheorie : Ansätze-Personen - Grundbegriffe, ed. par Ansgar Nünning, Stuttgart/ Weimar, 1998, pp. 112-113. Voir aussi en France les études ethnocritiques de J.-M. Privat, «Elias, Bakhtine et la littérature », in Norbert Elias et l'anthropologie, "Nous sommes tous si étranges... », S. Chevalier et J.-M. Privat (eds), Paris, CNRS Editions, coll. Anthropologie, 2004, pp. 185-195; M. Scarpa, "Le Ventre de Paris est-il un roman bien civilisé ? », in Norbert Elias et l'anthropologie, op. cit., pp. 196-203 et M.-Ch. Vinson, «La Civilisation des Robinsons », in J.-M. Privat (éd.), Ma Montagne. Lectures plurielles d'une robinsonnade pour la jeunesse, Recherches Textuelles, 6, Université Paul Verlaine - Metz, 2006, pp. 95116. Elias lui-même s'était inspiré de la littérature pour fonder puis pour illustrer ses théories. Voir par exemple le chapitre "Curialisation et romantisme aristocratique ", Norbert Elias, La Société de Cour, préface de Roger Chartier, Paris, 1985, pp. 239-305, où il décrit les rapports entre la curialisation de la noblesse, sa prédilection pour tout ce qui était nature (donc en opposition à ses contraintes) et les romans pastoraux tel que L'Astrée. - Il faut cependant signaler les études suivantes qui se réfèrent à Elias pour interpréter notamment La Princesse de Clèves : W. Matzat, « Affektrepräsentation im klassischen Diskurs : La Princesse de Clèves ", Französische Klassik. Theorie - Literatur - Malerei, ed. par Fritz Nies et Karlheinz Stierle, 1985, pp. 231-266 ; R. Galle, Geständnis und Subjektivität. Untersuchungen zum französischen Roman zwischen Klassik und Romantik, München 1986, pp. 13-54.

(3) R. Baader, « Mme de Lafayette in der Schule der Salons. Kasuistik und Geometrie in derPrincesse de 
$\mathrm{C}^{\prime}$ est à $\mathrm{M}^{\mathrm{me}}$ de Lafayette que revient le mérite d'avoir créé avec La Princesse de Clèves ${ }^{(4)}$ le prototype du roman psychologique de société. Ce «nouveau roman», par sa brièveté et par sa proximité spatio-temporelle surprit le public contemporain ${ }^{(5)}$ accoutumé au caractère volumineux des romans baroques ${ }^{(6)}$. Néanmoins l'intérêt des critiques se fixa plutôt sur la subtile observation et la description du monde intérieur, sur la conscience des personnages, domaine jusque-là largement inexploré dans la littérature.

Les hypothèses de N. Elias nous invitent à mettre en relation cette innovation littéraire avec le changement de structures sociales et psychiques. Sans vouloir avancer la thèse trop simpliste de l'œuvre comme reflet de la société ${ }^{(7)}$, il est cependant permis de supposer l'existence d'un rapport étroit entre la " psychologisation » inscrite dans $L a$ Princesse de Clèves et l'évolution de la société curiale. Les réflexions des moralistes du $\mathrm{XVII}^{\mathrm{e}}$ siècle nous serviront d'articulation entre la théorie socio-historique et l'œuvre littéraire. Nous pensons en effet que textes théoriques, œuvres des moralistes et corpus littéraire s'éclairciront réciproquement.

\section{La théorie d'Elias et la pensée moraliste du XVII' siècle}

La démarche interdisciplinaire d'Elias est caractérisée par les rapports étroits entre la sociologie et l'histoire culturelle d'une part, l'anthropologie et la psychologie d'autre part. Elle est illustrée par le sous-titre allemand de son œuvre principale, Über den Prozeß der Zivilisation ${ }^{(8)}$ et Soziogenetische und psychogenetische Untersuchun$\operatorname{gen}^{(9)}$. Le fait que soient traités dans un même livre les changements structuraux de la société et de l'individu reflète l'interdépendance que présuppose Elias entre ces deux pôles.

Comme on sait, la société de cour française occupe selon Elias une position exem-

Clèves », Cahiers d'Histoire des Littératures Romanes / Romanistische Zeitschrift für Literaturgeschichte 1995, 19, 1 : «C'est avec une certaine confusion que Fontenelle, en mai 1678, explique au Mercure Galant pourquoi il vient de relire pour la quatrième fois la Princesse de Clèves [...] Mme de Lafayette avait, elle-même, conseillé une lecture à plusieurs reprises " (traduit par nous-même, A.L.).

(4) $\quad \mathrm{M}^{\mathrm{me}}$ de Lafayette (1678), «La Princesse de Clèves », Romans et Nouvelles, éd. par Alain Niderst, Paris 1990, pp. 249-416 (dorénavant « $\mathrm{PdC} »)$.

(5) De la part des lecteurs contemporains, La Princesse de Clèves a également fait l'objet d'un certain nombre de critiques et surtout de Jean-Baptiste-Henry du Trousset de Valincour (1678), Lettres à Madame la Marquise de *** sur le sujet de "La Princesse de Clèves». La différence entre les " anciens » et les «nouveaux romans » a été traitée de manière moins polémique par le Chevalier Du Plaisir (1683), Sentiments sur les lettres et sur l'histoire avec des scrupules sur le style, éd. critique par Philippe Hourcade, Genève 1975, pp. 44-70. D'après lui, le nouveau roman se distingue entre autres par l'unité de l'action (p. 45), par le nombre restreint de personnages (p. 45) plus naturels (pp. 50 et suiv.), par son rapport à l'histoire récente (p. 45), par son refus de lieux trop éloignés (p. 46), par son respect de la vraisemblance (p. 46), et par son style d'impartialité (p. 56).

(6) Selon Du Plaisir (ibid., p. 44), le surgissement des nouveaux romans s'explique en partie par le fait qu'ils correspondent mieux au caractère national des Français que les genres littéraires antérieurs : «[...] c'est depuis peu seulement qu'on a inventé les nouvelles. Cette dernière espèce est principalement très convenable à l'humeur prompte et vive de notre Nation. Nous haïssons tout ce qui s'oppose à notre curiosité ».

(7) Elias conçoit les intellectuels d'une époque comme « les interprètes et les porte-paroles d'un "chœur social" " (id. . [1939], La Dynamique de l'Occident, traduit de l'allemand par Pierre Kamnitzer, Paris 1975, p. 266 [dorénavant Dynamique]). Voir aussi R. Wild, « Literatur und Zivilisationstheorie », op. cit., pp. 76-82.

(8) Traduit en français sous les titres La Civilisation des mœurs (t. 1) et La Dynamique de l'Occident (t. 2).

(9) Traduit littéralement : «Etudes sociogénétiques et psychogénétiques ». Pour ce qui est de 1'accueil de la pensée éliasienne dans les différentes sciences humaines, Karl-Siegbert Rehberg, Norbert Elias und die Menschenwissenschaften, Studien zur Entstehung und Wirkungsgeschichte seines Werkes, Frankfurt, 1996. 
plaire dans le processus de civilisation. Dès le début du XII siècle se dégagent des tendances centralisatrices qui s'accélèrent au tournant du XVI ${ }^{\mathrm{e}}$ siècle et aboutissent à l'Etat absolutiste de Louis XIV ${ }^{(10)}$. A époque médiévale, les terres de l'aristocratie militaire perdent en valeur en raison d'une circulation élargie de l'argent qui progressivement remplace l'économie du troc. Face à la bourgeoisie ascendante, les chevaliers nobles, dont les revenus nominaux stagnent, ont désormais du mal à maintenir leur position privilégiée tant sur le plan matériel que social ${ }^{(11)}$. Par contre, la bourgeoisie profite de l'augmentation de la masse monétaire et des flux financiers, tout comme le roi qui jouit d'un monopole fiscal. Grâce à sa supériorité financière qui lui permet d'entretenir une cour plus nombreuse, le seigneur central peut également renforcer sa position militaire. Il ne dépend plus des services d'aristocrates à rétribuer en terres mais peut financer une armée de soldats. Peu à peu, le roi monopolise ainsi la violence légitime ${ }^{(12)}$. Les guerriers nobles, dépourvus de leur fonction sociale, finissent par dépendre de leur suzerain aussi bien au niveau économique que militaire. Dans la deuxième moitié du XVII ${ }^{\mathrm{e}}$ siècle, les tendances centralisatrices s'imposent de sorte que la majorité de l'aristocratie se voit contrainte de rivaliser pour les faveurs du roi à la cour ${ }^{(13)}$. La cour est désormais la « configuration-clé » de la société : c'est le seul lieu qui puisse garantir aux nobles le prestige social nécessaire pour se distinguer de la bourgeoisie ainsi que leur survie matérielle (rentes, pensions, gratifications royales). Voilà deux motivations qui « enchâ̂nent par un double lien les membres des élites » ${ }^{(14)}$.

Cependant, la position du roi n'est pas non plus extérieure au réseau des interdépendances croissantes. Pour maintenir son pouvoir, le roi doit jouer sur clavier social composé pour l'essentiel de deux groupes ${ }^{(15)}$. Il revient au roi d'établir un équilibre - certes marqué de tensions - entre les bourgeois qui financent sa cour par leurs impôts et l'aristocratie qui garantit sa distinction sociale.

Une telle situation d'interdépendances et de rivalités des nobles pour les « chances que le monopolisateur veut bien mettre à leur disposition » ${ }^{(16)}$ entraîne des transformations collectives de comportement ${ }^{(17)}$. Pour amorcer une carrière à la cour ou pour maintenir une position de favori, les aristocrates doivent désormais veiller à se donner une réputation excellente. Il est ainsi hors de question pour eux de suivre leurs pulsions ou de régler leurs conflits par la simple violence physique ${ }^{(18)}$ :

Pour s'imposer, il faut cultiver d'autres qualités que celles qui assurent la victoire dans les passes d'armes : la réflexion, la prévision à long terme, la maîtrise de soi, la régulation rigoureuse de son émotivité, la connaissance du cœur humain et du champ social. ${ }^{(19)}$

Si les courtisans suivent, dans un premier temps, consciemment de telles contraintes, celles-ci s'intériorisent par la suite et finissent par faire partie intégrante de leur écono-

(10) N. Elias, Dynamique, pp. 29-183.

(11) N. Elias, ibid., p. 234.

(12) La proscription du duel par exemple date de 1630.

(13) N. Elias, ibid., pp. 225-240, désigne cette évolution par l'expression « la curialisation des guerriers"

(14) N. Elias, ibid., p. 237.

(15) N. Elias, ibid., p. 115 : «L'heure d'un pouvoir central fort dans une société à haut niveau de différenciation approche, quand l'ambivalence des intérêts des groupes fonctionnels les plus importants est si marquée, quand les centres de gravité se répartissent si également entre eux, qu'il ne peut y avoir, de quelque côté que ce soit, ni compromis, ni combats, ni victoire décisive.»

(16) N. Elias, ibid., p. 239.

(17) N. Elias, ibid., pp. 209-279.

(18) Voir également N. Elias, La Société de cour, Paris, Champs/Flammarion, 1985, p. 107 et suiv. (dorénavant Société).

(19) N. Elias, Dynamique, p. 242 
mie psychique ; elles forment ainsi une sorte de surmoi ou de conscience automati sée ${ }^{(20)}$. La régulation de la vie pulsionnelle par l'autocontrôle pouvait en revanche mener à une dissociation psychique, à une " "fêlure horizontale" qui traverse l'être dans toute sa largeur ${ }^{(21)}$, une déchirure de l'individu entre l'inconscient et le surmoi. Se développe ainsi la conception de l'homo clausus $^{(22)}$ : 1'homme renfermé sur lui-même, sur son monde intérieur, qu'il cache derrière une sorte de mur du monde extérieur de la société. Ce mur invisible est associé au corps en tant que « matérialisation des autocontraintes ", corps qui sépare l'intérieur de l'individu de sa face sociale ${ }^{(23)}$. L'idée d'une coexistence de deux réalités devient le paradigme de l'individu moderne, paradigme toujours actuel selon Elias. Pour s'approprier un comportement parfaitement adapté aux fonctions sociales de l'adulte, l'individu parcourt désormais une socialisation de plus en plus complexe et précoce que l'on peut comparer à un processus individuel de civilisation. Les différences manifestes entre les comportements se réduisent désormais à de fines nuances. La prévision ou l'anticipation des conséquences des actes individuels entraîne une «psychologisation » de l'idée que l'homme se fait de l'homme ${ }^{(24)}$.

Le XVII ${ }^{\mathrm{e}}$ siècle engendrera ainsi un idéal du comportement curial, la notion d'honnêteté $^{(25)}$. Le traité L'Honnête Homme ou l'Art de plaire à la Cour de Nicolas Faret, très connu à l'époque ${ }^{(26)}$, fait écho à $I l$ Cortegiano de Castiglione et devient la charte de ceux qui veulent réussir à la cour. Ils y trouvent des conseils pratiques et précis pour exceller dans tous les domaines de la vie sociale et morale. "La vertu n'est jamais oubliée ; c'est un homme de bien autant qu'un véritable homme de cour que veut former Faret ${ }^{(27)}$, commente René Bray. Dans la première moitié du siècle, un comportement dit « honnête» n'a apparemment pas encore été intériorisé par les courtisans. Il est donc nécessaire de le leur apprendre sous la forme de règles écrites. Une génération plus tard, le Chevalier de Méré présente un bilan plutôt descriptif de l'honnêteté ${ }^{(28)}$. S'appuyant sur les Essais de Montaigne, sa notion d'honnêteté comprend l'horreur du pédantisme bourgeois, le culte de la modération et la soumission à la raison. Selon Méré, l'honnête homme trouve son bonheur exclusivement à travers son existence sociale. Il sait se faire aimer, considère les sentiments des autres, supprime des affects violents et suit les normes de la bienséance. Pour ce qui est des qualités morales, il n'en a besoin qu'en fonction du commerce de la société. Les deux textes témoignent d'un glissement de sens,

(20) N. Elias, Dynamique, p. 200. Un tel passage témoigne de l'influence freudienne sur la pensée éliasienne. Voir Norbert Elias, Au-delà de Freud. Sociologie, psychologie, psychanalyse. Postface. Freud, Elias et la science de l'homme, par Bernard Lahire, Paris, La Découverte, coll. « Textes à l'appui », 2010.

(21) N. Elias, Dynamique, p. 204

(22) N. Elias, Société, p. 151. Sur la notion d'homo clausus, voir aussi l'article de J.-M. Privat, op. cit.

(23) N. Elias, „Einleitung“, Über den Prozeß der Zivilisation. Soziogenetische und psychogenetische Untersuchungen. Erster Band. Wandlungen des Verhaltens in den weltlichen Oberschichten des Abendlandes, pp. LXI-LXVI, 1939-1994. Cette introduction ne précède pas la traduction française du tome 1 (La Civilisation des mœurs).

(24) N. Elias, Dynamique, pp. $244 ; 246$.

(25) W. Matzat, op. cit., p. 238, fonde son étude de La Princesse de Clèves sur l'analyse des discours selon Foucault. Matzat établit une relation entre l'idéal de l'honnêteté et l'épistémè classique qui vise à une représentation transparente des signifiés par les signifiants. L'idéal de l'honnêteté essaie de modeler de façon esthétique et transparente un comportement naturel. Cette aspiration à la transparence des actes pose des problèmes dans la mesure où la vie sociale se concentre à la cour, problèmes qui seront repérés dans la critique des moralistes.

(26) Nicolas Faret (1630), L'Honnête Homme ou l'Art de plaire à la Cour, réimpr. Genève 1970. Cet ouvrage a été édité et réimprimé onze fois entre 1630 et 1681 et a ainsi eu plus de succès que d'autres traités poursuivant le même but.

(27) R. Bray, «Honnêteté », Dictionnaire des Lettres Françaises. Le dix-septième siècle, éd. par Georges Grente, Paris, 1954, p. 503.

(28) Chevalier de Méré, « De la vraie honnêteté », Euvres complètes, tome 3, éd. par Ch.-H. Boudhours, Paris 1930 , pp. 69-74; id.., «Suite de la vraie honnêteté », op. cit., tome 3, pp. 85-102. 
glissement qui s'effectue à deux niveaux : tout d'abord, « les qualités purement formelles prendront le pas sur la vraie vertu $»{ }^{(29)}$. Il ne s'agit donc plus de perfectionner le comportement de l'individu en tant que finalité pour soi, mais de l'adapter le plus parfaitement possible aux exigences de la vie en société, tout en dissimulant ses intérêts privés. Ensuite, le ton des traités évolue du texte prescriptif au bilan descriptif. Nous pouvons y voir un indice du fonctionnement de plus en plus inconscient des autocontraintes ${ }^{(30)}$.

Si le traité de Faret incite le courtisan à se construire une façade agréable, les essais, réflexions et maximes des moralistes empruntent la démarche inverse : ils visent à détruire cette surface pour découvrir ce qui se cache derrière ${ }^{(31)}$. La lucidité avec laquelle ils percent les fondements de la société semble anticiper les études d'Elias. Nous présenterons brièvement les approches moralistes de La Rochefoucauld et de Nicole, approches en quelque sorte complémentaires. Alors que le premier centre ses analyses plutôt sur l'individu ${ }^{(32)}$, le second démasque le fonctionnement de la société. Si les maximes de La Rochefoucauld témoignent donc des transformations relevant de la psychogénèse, les écrits de Nicole devancent les études d'Elias sur la sociogénèse.

A la cinquième et dernière édition des Maximes publiée de son vivant (1678), La Rochefoucauld fait précéder son ouvrage de l'épigraphe fameuses, «Nos vertus ne sont, le plus souvent, que des vices déguisés. » ${ }^{(33)}$ Cette devise indique que la préoccupation de l'auteur est de savoir montrer comment l'amour-propre, les passions, les humeurs et la fortune déterminent le comportement de l'individu, le plus souvent sans qu'il s'en rende compte ${ }^{(34)}$. C'est l'amour-propre, fil conducteur des Maximes, qui domine parmi les facteurs contraires à l'autonomie humaine. Dans la première édition (1665), le moraliste va jusqu'à consacrer une réflexion à ce phénomène ${ }^{(35)}$. Par le recours stylistique à la personnification, La Rochefoucauld montre le pouvoir et la dynamique intransigeante de l'amour-propre, "l'amour de soi-même, et de toutes choses pour soi » ${ }^{(36)}$ :

Rien n'est si impétueux que ses désirs, rien de si caché que ses desseins, rien de si habile que ses conduites; ses souplesses ne se peuvent représenter, ses transformations passent celles des métamorphoses, et ses raffinements ceux de la chimie. ${ }^{(37)}$

L'amour-propre est une instance étrangère et incompréhensible, qui fait cependant partie indestructible du moi et en tire sa dynamique ${ }^{(38)}$. La Rochefoucauld montre l'impossibilité de s'y soustraire et par conséquent l'impossibilité de l'autonomie humaine. Si l'on ne peut pas éliminer l'amour-propre, on est amené à le dissimuler, de moins en moins consciemment, sous la forme de quelque vertu : «Nous sommes si accoutumés à nous déguiser aux autres qu'enfin nous nous déguisons à nous-mêmes. » ${ }^{(39)}$

(29) R. Bray, op. cit., p. 502.

(30) Pour bien comprendre cette différence, il faut tenir compte du fait que Faret appartient à la bourgeoisie tandis que Méré est d'origine noble.

(31) P. Bénichou, Morales du grand siècle, Paris, Gallimard, 1948, pp. 118-127 et pp. 129-148.

(32) Surtout si l'on pense aux « Maximes », cependant un peu moins par rapport aux « Réflexions diverses ".

(33) F. de La Rochefoucauld (1665/1678), Maximes, suivies des Réflexions diverses, du Portrait de La Rochefoucauld par lui-même et des Remarques de Christine de Suède sur les Maximes, ed. par Jacques Truchet, Paris, 1992, p. 7.

(34) D. Steland, Moralistik und Erzählkunst von La Rochefoucauld und Mme de Lafayette bis Marivaux, München, 1984, p. 18 et suiv.

(35) La Rochefoucauld, op. cit., pp. 283-285.

(36) Ibid., p. 283.

(37) Id.., ibid.

(38) Vgl. K. Stierle (1985), « Die Modernität der französischen Klassik. Negative Anthropologie und funktionaler Stil », Französische Klassik, loc. cit. pp. 81-128, surtout p. 92 et suiv.

(39) La Rochefoucauld, op. cit., p. 33. 
Le lecteur d'Elias retrouve ici l'intériorisation des contraintes sociales qui ne seront désormais presque plus reconnaissables. La célèbre maxime «L'esprit est toujours la dupe du cœur » ${ }^{(40)}$ illustre comment l'homme se trompe doublement : il suit toujours les tentations de ses passions ou de l'amour-propre, mais il se croit néanmoins guidé par sa raison. La raison peut contribuer à sa propre défaite, parce que convaincue de se maîtriser ${ }^{(41)}$. La Rochefoucauld confronte ses contemporains à un miroir qui leur montre leurs vrais mobiles facilement oubliés grâce aux ruses intériorisées. Les réactions scandalisées aux Maximes démontrent jusqu'à quel point la régulation de la vie pulsionnelle est déjà automatisée à l'époque.

Dans son essai «De la charité et de l'amour-propre » $(1675)^{(42)}$, le janséniste Pierre Nicole traite l'amour-propre d'abord dans un contexte explicitement théologique pour le mettre ensuite en relation avec l'évolution de la vie sociale. Selon Nicole, tout homme aurait naturellement tendance à tyranniser les autres ${ }^{(43)}$, ce qui rendrait une vie en société inconcevable. Cependant, la peur de mourir et le désir de sûreté mènent l'homme à vivre en société. Il paie cette existence confortable avec la maîtrise de son amour-propre et le renoncement à la violence physique. L'amour-propre apprivoisé se voit contraint à satisfaire ses besoins - principalement celui d'être reconnu et aimé $^{(44)}$-, par d'autres stratégies comme, par exemple, la civilité.

Ainsi, se voyant exclus de la violence ouverte, ils [les hommes] sont réduits à chercher d'autres voies, et à substituer l'artifice à la force, et ils n'en trouvent point d'autre que de tâcher de contenter l'amour-propre de ceux dont ils ont besoin, au lieu de le tyranniser ${ }^{(45)}$.

S'établit, par la suite, une sorte de commerce d'assiduités et de civilités selon le principe «On donne pour obtenir. » ${ }^{(46)}$ L'amour-propre se cache sous le masque de l'honnêteté qui n'est qu' « un amour-propre plus adroit que celui du commun du monde » ${ }^{(47)}$. L'anticipation des conséquences potentielles du propre comportement détermine les actions des courtisans ${ }^{(48)}$. Nicole décrit avec quelque fascination comment un tel « amour-propre éclairé », bien que théologiquement condamnable, peut faire fonctionner la vie en société :

Quelque corrompue que cette société fût au dedans et aux yeux de Dieu, il n'y aurait rien au-dehors de mieux réglé, de plus civil, de plus juste, de plus pacifique, de plus honnête, de plus généreux ; et ce qui serait de plus admirable, c'est que, n'étant animé et remué que par l'amour-propre, l'amour-propre n'y paraîtrait point, et qu'étant entièrement vide de charité, on ne verrait partout que la forme et les caractères de la charité. ${ }^{(49)}$

(40) Ibid.., p. 29.

(41) D. Steland, op. cit., pp. 14 et suiv., qui cite l'interprétation de $\mathrm{M}^{\mathrm{me}}$ de Schomberg.

(42) Pierre Nicole [1675], « De la charité et de l'amour-propre », Euvres philosophiques et morales, éd. par Charles Marie Gabriel Bréchillet Jourdain, réimpr. de l'éd. Paris 1845, Hildesheim 1970, pp. 179-206.

(43) P. Nicole, op. cit., p. 179 et suiv. : "Cette disposition tyrannique [i.e. l'amour-propre] étant empreinte dans le fond du cœur de tous les hommes, les rend violents, injustes, cruels, ambitieux, flatteurs, envieux, insolents, querelleurs.» Voir aussi La Rochefoucauld, op. cit., p. 283 : «il [1'amourpropre] rend les hommes idolâtres d'eux-mêmes, et les rendrait les tyrans des autres si la fortune leur en donnait les moyens. »

(44) P. Nicole, op. cit., pp. 183-184: «Que la plus générale inclination qui naisse de l'amour-propre est le désir d'être aimé ».

(45) Ibid., p. 181.

(46) Ibid., p. 81.

(47) Ibid., p. 185.

(48) D. Steland, p. 47 : «L'amour-propre agit donc seulement après une pause de réflexion, pendant laquelle elle gagne un savoir qui pénètre les attentes de l'autre » (traduit par nous-même, A.L.)

(49) P. Nicole, op. cit., p. 200. 
La Rochefoucauld et Nicole rendent compte de deux façons différentes du processus de civilisation. Le premier ramène le comportement des courtisans à son origine, le second, plus éliasien, décrit plutôt comment il s'est développé à partir de cette même origine.

Rappelons enfin que pendant longtemps les critiques ont discuté de l'identité de l'auteur de La Princesse de Clèves, possible fruit aussi d'une collaboration entre La Rochefoucauld et $\mathrm{M}^{\mathrm{me}}$ de Lafayette ${ }^{(50)}$. La vision de l'homme communiquée par le roman est en effet proche de celle du moraliste ${ }^{(51)}$. Tandis que La Rochefoucauld décrit l'aliénation du moi sous forme plutôt abstraite, $\mathrm{M}^{\mathrm{me}}$ de Lafayette en fait avec La Princesse de Clèves un cas concret, à la manière des jeux d'esprit dans les salons littéraires qu'elle fréquente ${ }^{(52)}$.

\section{La Princesse de Clèves et l'anticipation du moi-sujet}

Selon R. Wild ${ }^{(53)}$, le rôle que joue l'œuvre littéraire dans le processus de civilisation s'actualise au cours de la lecture et s'organise autour de deux axes principaux : en premier lieu, la littérature exprime les conditions sociales et psychiques de son époque, donc de la phase de l'évolution civilisatrice atteinte à tel moment historique. En second lieu, l'œuvre propose une réflexion critique sur ce processus de civilisation ${ }^{(54)}$. Pour préciser de quelle manière La Princesse de Clèves s'inscrit dans le processus de civilisation, nous tiendront compte de ces deux types de fonctions interdépendantes de l'œuvre littéraire présentées par Wild.

Examinons d'abord en quoi le roman de $\mathrm{M}^{\mathrm{me}}$ de Lafayette remplit des « fonctions stabilisantes $»{ }^{(55)}$. Distinguons avec Wild quatre aspects différents qui peuvent cependant interférer ${ }^{(56)}$.

En premier lieu, il s'agit de la «fonction didactique » qui consiste à présenter sous la forme de caractères fictifs le comportement exigé par la civilisation et inciter les lecteurs à s'y conformer. Cette fonction de la littérature gagne d'autant plus en importance que le processus de civilisation évolue et que la socialisation de l'individu devient de plus en plus complexe ${ }^{(57)}$. En second lieu, la « fonction sociale et sensibilisante » sert à soutenir l'auto-régularisation du comportement et le changement correspondant de l'économie psychique. Le texte littéraire inviterait ainsi son destinataire non seulement à la lecture et à la conversation qui peut s'en suivre, mais aussi l'inciterait à s'entraîner à l'analyse du comportement social de personnages fictifs. En troisième lieu, la « fonction compensatrice » permet de relâcher dans la fiction les contraintes imposées par la

(50) De nos jours, on ne lui conteste plus l'attribution de son œuvre. (R. Baader, « Madame de Lafayette, La Princesse de Clèves [1678] », 17. Jahrhundert. Roman, Fabel, Maxime, Brief, Tübingen, 1999, p. 244).

(51) K. Stierle, op. cit., p. 113 : «Ce roman met en mouvement les Maximes et réflexions. » (traduit par nous-même, A.L.). Bien que $\mathrm{M}^{\mathrm{me}}$ de Lafayette ait préféré les maximes plus optimistes de $\mathrm{M}^{\mathrm{me}} \mathrm{de} \mathrm{Sa}^{-}$ blé à celles de son ami La Rochefoucauld elle demande plus tard au duc d'introduire une de ses propres maximes parmi les siennes, ce qu'il fait (R. Baader, op. cit., p. 249).

(52) R. Baader, op. cit., p. 246.

(53) R. Wild, Literatur im Prozeß der Zivilisation. Entwurf einer theoretischen Grundlegung der Literaturwissenschaft, Stuttgart, 1982.

(54) R. Wild, « Literatur und Zivilisationstheorie», op. cit., p. 79.

(55) R. Wild, Literatur im Prozeß der Zivilisation, pp. 73-117 et "Literatur und Zivilisationstheorie ", op. cit., p. 78 et suiv.

(56) C'est pourquoi elles ne seront pas traitées de façon analytique dans l'interprétation qui s'ensuit.

(57) Cette fonction prédomine dans le roman d'éducation (R. Wild, «Literatur und Zivilisationstheorie », op. cit., p. 77). 
civilisation en représentant par exemple mille et une formes de violence ouverte. La littérature documente ainsi l'ambivalence du processus civilisateur : si la société pacifiée tend à supprimer l'agressivité humaine, elle ne saurait pour autant l'éliminer. En dernier lieu, la « fonction élargissante » naîtrait du désir d'informer sur des manières de vivre et de se comporter non accessibles dans la vie quotidienne des lecteurs. Cette fonction serait surtout remplie par les récits autobiographiques de voyage qui décrivent la réaction d'un individu à un monde étranger. Par cet ensemble de " fonctions stabilisantes », la littérature tend à participer activement et à sa façon au processus de civilisation : elle le reflète et l'aide à s'imposer.

L'œuvre qui fait centralement ou latéralement du processus de civilisation son sujet peut en fait s'y opposer de façon critique, frontalement ou sur un mode plus sublimi nal $^{(58)}$. Après la conformation la confrontation possible donc.

Dans ce deuxième groupe Wild nomme d'abord la « fonction critique » qui met en question le processus civilisateur par la présentation de comportements qui ne sont pas, plus, ou pas encore en accord avec le niveau de civilisation atteint. La « fonction anticipative » s'y trouve étroitement liée ; elle consiste à présenter des façons d'agir progressistes qui se réalisent soit uniquement au moment de la lecture, dans l'imagination, ou bien véritablement, dans une époque à venir - mais qui, en tout cas, contrastent avec le comportement dominant de l'époque.

\section{1. «Une parfaite imitation du monde de la Cour» : les fonctions stabilisantes}

Contrairement au roman baroque, La Princesse de Clèves renonce aux déplacements incessants dans l'espace. La narratrice se borne à deux lieux d'action : la cour d'Henri II dans les dernières années de son règne ${ }^{(59)}$ et, par moments, Coulommiers, la maison de campagne des Clèves. Cette concentration spatiale répond aux tendances centralisatrices mentionnées plus haut. Elle est poussée si loin que le lecteur d'aujourd'hui confronté à ce « huis clos curial » ${ }^{(60)}$ et conséquemment à l'impossibilité quasi absolue de retrait privé - « il était difficile d'avoir une conversation particulière » $(\mathrm{PdC}, 270)-$ éprouve un certain malaise existentiel.

C'est surtout la protagoniste qui verra diminuer sa liberté par des contraintes spatiales. Ainsi, pour que la princesse puisse se retirer une première fois à Coulommiers, il faut à la narratrice une raison aussi grave que le deuil de $\mathrm{M}^{\mathrm{me}}$ de Chartres. M. de Clèves n'attend cependant pas longtemps avant de rappeler à sa femme son obligation de réapparaître à la Cour ${ }^{(61)}$. Et lorsque, plus tard, la princesse éprouve à nouveau le désir de partir pour la campagne, cette fois pour se mettre à l'abri d'une éventuelle découverte de ses sentiments pour M. de Nemours, les règles du jeu social sont claires : «Elle n'était pas maîtresse de s'éloigner » $(\mathrm{PdC}, 319)$. Les forces centripètes de cette société, représentées surtout par le mari, ne lui permettent pas de choisir son lieu de séjour. Ainsi, obligée de rester présente à la cour, $\mathrm{M}^{\mathrm{me}}$ de Clèves est à tout moment un personnage public. Même sa chambre, lieu extrêmement privé dans la conception du lecteur d'au-

(58) R. Wild, Literatur im Prozeß der Zivilisation, op. cit., pp. 118-137 et « Literatur und Zivilisationstheorie ", op. cit., p. 81 et suiv.

(59) Pourtant cette cour ressemble beaucoup à celle de Louis XIV, comme les critiques n'arrêtent pas de souligner.

(60) N. Grande, Stratégies de romancières. De "Clélie » à "La Princesse de Clèves », Paris, Champion, 1999 , p. 133 et p. 134 : La cour « exerce un rôle actif en figurant la pression sociale qui s'exerce sur la femme. En cela elle apparaît emblématique de toutes les formes de contraintes sociales qui s'exercent sur les femmes... et aussi sur les hommes. »

(61) PdC, $303:$ : Il est temps que vous voy[i]ez le monde, et que vous receviez ce nombre infini de visites dont aussi bien vous ne sauriez vous dispenser. » Pour d'autres passages semblables, PdC, 349, et 356 : «il [M. de Clèves] voulait absolument qu'elle revînt à Paris. Elle y revint comme il ordonnait » 
jourd'hui, est, malgré elle, accessible aux visiteurs : le duc de Nemours, que la princesse refuse d'accueillir de bon gré, s'adresse à son mari qui « le mena à l'heure même dans la chambre de sa femme » $(\mathrm{PdC}, 340)$. Elle en éprouve du «trouble » et de l'«étonnement ». Au rétrécissement de l'espace s'ajoute dans de telles scènes une dimension relativement négligée par Elias dans les travaux cités : la femme, par rapport aux courtisans masculins, dispose d'un espace d'action encore plus restreint et surveillé ${ }^{(62)}$.

Pendant un certain temps, $\mathrm{M}^{\mathrm{me}}$ de Clèves espère trouver à Coulommiers un lieu de repos personnel et de retraite privé, par opposition au caractère public (et officiel) de la cour. Pourtant, il lui est impossible de se retirer véritablement, de se dérober à la vue des autres : à plusieurs reprises, Nemours trouve le moyen d'y pénétrer sans qu'elle le désire, voire même sans qu'elle le sache ${ }^{(63)}$. Le seul moyen de se soustraire au regard scrutateur des autres est de traduire son incompréhensible « goût pour la solitude " $(\mathrm{PdC}$, 349) par des malaises et des maladies feintes. $\mathrm{M}^{\mathrm{me}}$ de Clèves a souvent recours à ce remède, à ce faux fuyant ${ }^{(64)}$. Mais chaque fois, «le monde » observera attentivement la «malade imaginaire » pour découvrir le véritable motif de sa retraite, sa désocialisation comme on pourrait dire ${ }^{(65)}$.

La cour décrite par $\mathrm{M}^{\mathrm{me}}$ de Lafayette (et par N. Elias) se caractérise par un réseau d'interdépendances. Les courtisans dépendent avant tout du roi, ce qui les fait lutter pour l'acquisition et le maintient des faveurs royales. Même si le roi n'est pas absolument le seigneur absolu ${ }^{(66)}$ :

Le roi se reposait sur lui [le connétable de Montmorency] de la plus grande partie du gouvernement des affaires et traitait le duc de Guise et le maréchal de Saint-André comme ses favoris ; mais ceux que la faveur ou les affaires approchaient de sa personne, ne s'y pouvaient maintenir qu'en se soumettant à la duchesse de Valentinois ; et, quoiqu'elle n'eût plus de jeunesse ni de beauté, elle le gouvernait avec un empire si absolu que l'on peut dire qu'elle était maîtresse de sa personne et de l'État ${ }^{(67)}$.

A la suite de la mort du roi, " la cour changea entièrement de face " $(\mathrm{PdC}, 377)$ et l'empire de Diane de Poitiers s'écroule du jour au lendemain. On retrouve très nettement cette « distinction par la dépendance » ${ }^{(68)}$ qu’Elias juge caractéristique de la société de cour. Le cas du vidame de Chartres en fournit un exemple représentatif: si la reine a fait de lui son confident unique ${ }^{(69)}$, elle le perd dès qu'elle le soupçonne de la tromper.

La réduction spatiale entraîne comme deuxième conséquence une concentration de

(62) M. Danahy, The feminization of the novel, Gainsville, 1991, pp. 101-108. Voir également K. Glosch et Ch. Losfeld, "Pavillons und Palisade. Geschlechterorte in Mme de La Fayettes La Princesse de Clèves », in : Geschlechterdifferenzen. Beiträge zum 14. Nachwuchskolloquium der Romanistik, Bonn, 1999, p. 44.

(63) H. Merlin, Public et Littérature en France au XVII siècle, Paris, 1994, p. 336 : «...l'histoire de la princesse de Clèves peut se lire comme la quête vaine d'un lieu soustrait au public.»

(64) PdC, 286 (avant le bal du maréchal Saint André), 324 et 327 (avant et après la lecture de la lettre).

(65) PdC, 357 et 375. Voir également H. Merlin,op. cit., p. 323 : «Sous l'empire de l'émotion, les personnages [...] se retirent chez eux. [...] Mais leur retraite n'a encore rien d'un espace privé puisque, pour rester seuls, ils doivent simuler la maladie. »

(66) Mais c'est lui seul qui détient les chances matérielles dont il peut faire profiter ses favoris. Pdc, 258 : «La libéralité du roi fournissait à cette dépense ; ce prince allait jusqu'à la prodigalité pour ceux qu'il aimait. "

(67) PdC, 256. Voir aussi la dépendance réciproque entre le roi et sa maîtresse : «Il [le roi] vient de rentrer chez lui très affligé, mais je ne sais s'il l'est davantage de l'opinion que Mme de Valentinois a sacrifié sa bague que de la crainte de lui avoir déplu par sa colère. » $(\mathrm{PdC}, 296)$

(68) Sur ce point d'équilibre instable comme économie propre à la logique curiale et aux positionnements structuraux des acteurs se reporter à la très éclairante préface de R. Chartier, "Formation sociale et économie psychique : la société de cour dans le procès de civilisation », Elias, La Société de cour,op. cit., pp. I-XXVIII.

(69) $\mathrm{PdC}, 334$ : « elle eût en moi une entière confiance ; elle ne fit plus rien sans m'en parler » 
1'intrigue ${ }^{(70)}$. La narratrice de La Princesse de Clèves ne met pas en scène une aventure héroïque extérieure, mais excelle dans la présentation de la vie intérieure des personnages $^{(71)}$. Ce glissement du centre d'intérêt souligne l'importance que revêt l'observation de soi-même et d'autrui pour survivre à la cour. Puisque la bienséance contraint l'individu à se comporter selon les normes de l'honnêteté et à dissimuler ses véritables sentiments, il devient de plus en plus difficile de connaître - à partir des nuances de leur comportement - les vrais motifs des autres. Cette évolution a son impact sur la littérature, comme N. Elias le note expressément :

Les indices de cette transformation ne se trouvent pas seulement dans les manuels du savoir-vivre ; les ouvrages littéraires destinés à divertir la bonne société en font également état. L'observation des hommes que la vie de la cour rend nécessaire se reflète dans 1'art du portrait littéraire ${ }^{(72)}$.

C'est encore le vidame de Chartres qui peut servir d'exemple de cette «psychologisation » sociale. Après que la reine lui a présenté les conditions liées à l'offre de devenir son confident préféré, il fait preuve de son savoir psychologique et de sa capacité de prévoir les prolongements futurs de ses actes - sans qu'il puisse pour autant en profiter pour modeler son comportement :

Les deux jours qu'elle m'avait donnés pour y penser ne me parurent pas trop longs pour me déterminer. Je voyais qu'elle voulait savoir si j'étais amoureux, et qu'elle ne souhaitait pas que je le fusse. Je voyais les suites et les conséquences du parti que j'allais prendre, ma vanité n'était pas peu flattée d'une liaison particulière avec une reine [...]. D'autre côté, j'aimais Mme de Thémines et, [...], je ne me pouvais résoudre à rompre avec elle. Je voyais aussi le péril où je m'exposais en trompant la reine et combien il était difficile de la tromper ; néanmoins, je ne pus me résoudre à refuser ce que la fortune m'offrait, [...] (PdC, 332).

Les courtisans, se voyant exposés à l'observation infatigable et incessante de leurs pairs, maîtrisent leurs pulsions et cachent leur vie intérieure pour ne pas rendre visible quelque mouvement spontané du cœur. N. Elias a décrit plus largement comment, dès la Renaissance, les hommes commencent à se concevoir en tant que homines clausi. Il cite, lui-même, un exemple tiré de La Princesse de Clèves ${ }^{(73)}$ : après l'aveu extraordinaire de ses sentiments pour un autre homme, $\mathrm{M}^{\mathrm{me}}$ de Clèves demande à son mari de la retirer de la cour pour la protéger de ses propres passions. Cependant, M. de Clèves, comptant sur la maîtrise intériorisée des pulsions de sa femme, ne juge pas nécessaire de la contrôler.

[...] vous avez plus de force et plus de vertu que vous ne pensez. [...] De l'humeur dont vous êtes, en vous laissant votre liberté, je vous donne des bornes plus étroites que je ne pourrais vous en prescrire. $(\mathrm{PdC}, 356)$

(70) Valincour, op. cit., pp. 19-23, préfère une restriction encore plus sévère. Il traite de « digressions »le discours que $\mathrm{M}^{\mathrm{me}}$ de Chartres tiendra à sa fille ainsi que les histoires de $\mathrm{M}^{\mathrm{me}}$ de Tournon ou d'Anne de Boylen. Pourtant, ces passages sont justifiables par leur fonction pour «l'éducation sentimentale » de la protagoniste; ils « forment autant de leçons destinées à apprendre à Mme de Clèves la cour dans sa vérité »(H. Merlin, op. cit., p. 327 et suiv.).

(71) Du Plaisir, op. cit., p. 50, voit derrière ce changement de goût l'envie des lecteurs de s'identifier aux personnages, jugement qui affirme les fonctions stabilisantes assumées par un roman tel que $L a$ Princesse de Clèves.

(72) N. Elias, Dynamique, p. 247. Du Plaisir, op. cit., p. 52, souligne la difficulté de cette introspection: d'une part, il s'agit de matières « imperceptibles », d'autre part, l'anatomie du cœur humain est une science relativement nouvelle à qui manque de notions précises. Il s'agit « des choses qui par le peu de connaissance qu' on en a eu jusqu'ici, n'ont presque point encore de termes propres. »

(73) N. Elias (1939), La civilisation des mours, Paris, Calmann-Lévy, 1973. 
Malgré le tableau idéalisant que $\mathrm{M}^{\text {me }}$ de Lafayette peint dans les premières pages du roman, la cour se révèle très vite être contaminée par les intrigues des maîtresses et l'opportunisme des favoris :

L'ambition et la galanterie étaient l'âme de cette cour, et occupaient également hommes et femmes. Il y avait tant d'intérêts et tant de cabales différentes, et les dames y avaient tant de part que l'amour était toujours mêlé aux affaires et les affaires à l'amour. Personne n'était tranquille, ni indifférent ; on songeait à s'élever, à plaire, à servir ou à nuire ; on ne connaissait ni l'ennui, ni l'oisiveté, et on était toujours occupé des plaisirs ou des intrigues ${ }^{(74)}$.

Si la narratrice tient ainsi à mettre en garde le lecteur de se méfier des apparences ${ }^{(75)}$, $\mathrm{M}^{\mathrm{me}}$ de Chartres agit de même pour prévenir sa fille du «peu de sincérité des hommes, leur tromperies et leur infidélités » $(\mathrm{PdC}, 260)$ : "Si vous jugez des apparences en ce lieu-ci, [...], vous serez souvent trompée : ce qui paraît n'est jamais la vérité » (PdC, 277). Ses propos se révèlent ironiquement d'autant plus vrais que $\mathrm{M}^{\mathrm{me}}$ de Chartres donne elle-même un exemple de cette hypocrisie dont elle prétend protéger sa fille ${ }^{(76)}$. Visant à obtenir une preuve de la passion de $\mathrm{M}^{\mathrm{me}}$ de Clèves pour Nemours, la mère raconte de fausses rumeurs à sa fille pour exciter sa jalousie. Ce n'est d'ailleurs pas le seul moment où le lecteur a lieu de douter de la sincérité de son amour maternel ${ }^{(77)}$. L'histoire intercalée de $\mathrm{M}^{\mathrm{me}}$ de Tournon révèle une femme qui pousse à l'extrême le jeu hypocrite. Sa manipulation de Sancerre et d'Estouteville qui lui sont tous les deux dévoués montre une perfection tactique qui fait frissonner M. de Clèves, pourtant plus longtemps à la cour que sa femme : «L'adresse et la dissimulation [...] ne peuvent aller plus loin qu'elle les a portées » $(\mathrm{PdC}, 303)$.

$\mathrm{M}^{\mathrm{me}}$ de Lafayette, en assurant que son œuvre est « une parfaite imitation du monde de la court [sic] et de la manière dont on y vit ${ }^{(78)}$, répond d'une relation mimétique de son roman avec la société curiale. Comme on vient de le constater, le lecteur d'Elias trouve, dans la description de la cour et du comportement des personnages, une affirmation de la poussée civilisatrice, de la transformation de contraintes sociales en autocontraintes. Le mépris avec lequel M. de Clèves condamne le comportement de $\mathrm{M}^{\text {me }}$ de Tournon fait cependant douter du fait que le roman ne reflète pas seulement une certaine époque du processus de la civilisation, mais aussi, tout à la façon des moralistes, sa critique.

\section{Fonctions réflexives : critique et anticipation}

$\mathrm{Si} \mathrm{M}^{\mathrm{me}}$ de Chartres, se doutant de l'inclination secrète de sa fille, lui conseille de se retirer de la cour, elle fait allusion à l'insuffisante « éducation sentimentale » de la princesse et se fait ainsi porte-parole de la fonction critique du roman. Aux yeux de sa mère, $\mathrm{M}^{\mathrm{me}}$ de Clèves n'est pas encore assez maîtresse d'elle-même pour contrôler sa passion et pour éviter le danger de «tomber comme les autres femmes » (PdC, 291). La narra-

(74) PdC, 264. Voir aussi PdC, 375 : «Une cour, aussi partagée et aussi remplie d'intérêts opposés, n’était pas dans une médiocre agitation à la veille d'un si grand événement ; néanmoins, tous les mouvements étaient cachés, et l'on ne paraissait occupé que de l'unique inquiétude de la santé du roi. »

(75) PdC, 253 et suiv. : « [...] il semblait qu'elle [la reine] souffrît sans peine l'attachement du roi pour la duchesse de Valentinois, et elle n'en témoignait aucune jalousie, mais elle avait une si profonde dissimulation qu'il était difficile de juger de ces sentiments, et la politique l'obligeait d'approcher cette duchesse de sa personne [...]» (c'est nous qui soulignons, A.L.). Pour ce qui est des vrais sentiments de la reine qui représente de manière parfaite la maîtrise des pulsions, voir PdC, 334 .

(76) R. Baader, op. cit., p. 252.

(77) D'ailleurs, M. de Clèves procède de façon comparable pour savoir qui est le bien-aimé de la princesse $(\mathrm{PdC}, 358)$.

(78) Jean de Bazin, Lettres de Madame de La Fayette au Chevalier de Lescheraine. Texte provenant des Archives de Turin, Paris, 1970, p. 1 (Lettre du 13 avril 1678). 
trice du roman nous montre, d'un coté, le niveau d'autocontrainte atteint par le processus de civilisation, mais représente en même temps la maîtrise des pulsions de la protagoniste en état de naître et de s'accomplir ${ }^{(79)}$.

Au moment de son entrée dans le monde, $\mathrm{M}^{1 \mathrm{le}}$ de Chartres, âgée seulement de quinze ans, ne partage pas encore entièrement ce savoir psychologique qui distingue les autres membres de la société de cour. Ainsi, quand M. de Clèves se plaint de ne pas recevoir de la part de sa femme les signes témoins d'une vraie inclination, elle répond ingénument que c'est par bienséance qu'elle n'ose pas faire paraître sa passion. De même, la princesse interprète le fait de rougir à la vue de son mari comme signe de joie, jusqu'à ce que celui-ci la détrompe : ce n'est que sa modestie. La narratrice de commenter alors : « $\mathrm{M}^{\text {lle }}$ de Chartres ne savait que répondre, et ces distinctions étaient au-dessus de ces connaissances » $(\mathrm{PdC}, 271)$. Si les méprises citées restent sans suites dangereuses pour la princesse, il n'en est pas de même pour d'autres qui suivront.

Lorsque, par exemple, la princesse de Clèves est invitée à un bal auquel le duc de Nemours ne peut participer - il trouverait insupportable l'idée que sa maîtresse aille à un bal sans qu'il puisse y être présent - elle décide de rester chez elle. La conscience de la princesse, bien que soupçonnant le vrai mobile de son refus, arrive à le remplacer par un argument spécieux : elle croit ne pas pouvoir aller chez le Maréchal Saint-André parce que celui-ci l'aime et qu'elle doit donc l'éviter. Il revient, une fois de plus, à la narratrice de dévoiler la dissimulation : « $\left[\mathrm{M}^{\mathrm{me}}\right.$ de Clèves] fut bien aise d'avoir une raison de sévérité pour faire une chose qui était une faveur pour M. de Nemours » (PdC, 286). Au niveau de 1'histoire, c'est la reine dauphine qui serait presque arrivée à démêler la vraie motivation de la princesse si $\mathrm{M}^{\mathrm{me}}$ de Chartres, par sa présence d'esprit, ne l'avait sauvée ${ }^{(80)}$.

La princesse est bien consciente du fait qu'elle ne parvient pas encore à se maîtriser parfaitement. C'est pourquoi elle éprouve le besoin d'être protégée de sa propre faiblesse par d'autres personnages, d'abord par sa mère, puis par son mari ${ }^{(81)}$. Après avoir appris l'histoire de $\mathrm{M}^{\mathrm{me}}$ de Tournon, elle se croit pourtant revenue de sa passion adultère par ce dangereux exemple et elle est d'accord pour rentrer à Paris - à tort, comme elle doit reconnaître le soir-même : «Elle connut bien qu'elle s'était trompée lorsqu'elle avait cru n'avoir plus que de l'indifférence pour M. de Nemours » $(\mathrm{PdC}, 309)^{(82)}$. C'est le décalage entre le savoir psychologique approfondi du duc de Nemours, courtisan parfait, et celui de la jeune princesse, encore apprentie dans ce domaine, qui rend leur inclination réciproque si dangereuse pour elle :

Quelque application qu'elle eût à éviter ses regards et à lui parler moins qu'à un autre, il lui échappait de certaines choses qui partait d'un premier mouvement, qui faisait juger à ce prince qu'il ne lui était pas indifférent. Un homme moins pénétrant que lui ne s'en fût peut-être pas aperçu, mais il avait déjà aimé tant de fois qu'il était difficile qu'il ne connut pas quand on l'aimait (PdC, 313).

Puisque ce manque de connaissance du cœur humain et de soi-même va de pair avec une certaine maladresse dans la dissimulation de ses sentiments, $\mathrm{M}^{\mathrm{me}}$ de Clèves se trahit à plusieurs reprises tant par la parole que par son comportement.

(79) Si l'héroïne n'est pas la seule à faire remarquer des ruptures avec l'habitus dominant de la société de cour, elle en fournit pourtant les exemples les plus représentatifs. Il est un autre personnage qui fait voir, par moments, un comportement en désaccord avec le «niveau de civilisation atteint »; c'est le chevalier de Guise, qui se montre en public, à plusieurs reprises, amoureux de $\mathrm{M}^{11 \mathrm{e}}$ de Chartres $(\mathrm{PdC}$, 265 et 272).

(80) Toutefois, la princesse ne peut s'empêcher de rougir, et le duc de Nemours s'en rend bien compte.

(81) «Elle se trouvait malheureuse d'être abandonnée à elle-même, dans un temps où elle était si peu maîtresse de ses sentiments et où elle eût tant souhaité d'avoir quelqu'un qui pût la plaindre et lui donner de la force. » $(\mathrm{PdC}, 292)$

(82) Lors de l'épisode de la lettre, ayant fait l'expérience douloureuse et humiliante de la jalousie, elle se croit, encore une fois, «entièrement guérie de l'inclination qu'elle avait pour ce prince » $(\mathrm{PdC}, 327)$. 
Son premier bal fera ainsi connaître à la princesse combien il est important de bien peser ses paroles dans la société de cour. Le roi et la reine ont provoqué une danse de M. de Nemours avec cette belle personne ; ils demandent ensuite à l'un et à l'autre s'ils soupçonnent avec qui ils viennent de danser. Si le duc ne nie pas connaître l'identité de sa partenaire, cette dernière prétend ne pas savoir ou pouvoir deviner qui il est. Cependant, elle n'arrive pas à tromper la reine qui réplique : « [...] il y a même quelque chose d'obligeant pour $M$. de Nemours à ne vouloir pas avouer que vous le connaissez sans l'avoir jamais vu » $(\mathrm{PdC}, 275)$. La princesse apprend ainsi qu'elle ne pèse pas assez ses paroles, et qu'elle n'arrive pas encore à prévoir leurs prolongements. De même, quand elle se précipite sans réfléchir dans la chambre de sa mère après la soirée pour lui raconter ce qu'elle a vécu, bien qu'il soit déjà très tard, elle trahit son émoi causé par la première rencontre avec le duc de Nemours ${ }^{(83)}$.

L'héroïne se trahit par la parole mais aussi par son comportement. A travers des signes physiques, elle révèle involontairement sa passion pour Nemours. La description d'un accident par lequel Nemours tombe du cheval représente un des passages-clés dans ce contexte :

L'intérêt qu'elle y prenait lui donna une appréhension et un trouble qu'elle ne songea pas à cacher; elle s'approcha de lui avec les reines et, avec un visage si changé qu'un homme moins intéresse que le chevalier de Guise s'en fût aperçu (PdC, 321. C'est nous qui soulignons, A.L.).

Pas seulement le chevalier de Guise, mais aussi le duc de Nemours s'en rend infailliblement compte en rouvrant les yeux; et $\mathrm{M}^{\mathrm{me}}$ de Clèves s'en voudra terriblement de lui avoir révélé son secret le plus intime. Cet incident, comme d'autres ${ }^{(84)}$, prouvent à la princesse que sa passion se situe au-delà (ou en deçà) de sa conscience et de sa volonté.

Après ces transgressions plus ou moins dangereuses, l'aveu que $\mathrm{M}^{\mathrm{me}}$ de Clèves fait de son inclination à son mari représente sans aucun doute la plus grave transgression verbale des règles de la bienséance, et surtout, dans la critique de l'époque, de la vraisemblance. L'héroïne elle-même est bien consciente de l'extraordinaire de son acte. Façonnant le premier projet d'aveu, « elle y trouva de la folie » ${ }^{(85)}$; pourtant, elle finit par s'y résoudre.

Dans la perspective éliasienne, il est intéressant de remarquer lors de l'aveu à son mari la différence que fait la princesse entre ses sentiments par lesquels elle admet lui déplaire et ses actions par lesquelles elle lui promet de ne pas fauter. C'est la conception même de l'homo clausus et de son espace intérieur. Les réactions des autres personnages du roman soulignent d'ailleurs combien la vérité intérieure de la princesse - par son expression sincère - sort du convenable dans une société où la communication directe, surtout si elle a pour sujet des passions (et des corps), est impossible ${ }^{(86)}$. La reine dau-

(83) PdC, 279. Peu après, se doutant de son inclination pour Nemours, « elle ne trouva plus la même disposition à dire à sa mère ce qu'elle pensait des sentiments de ce prince qu'elle avait eue à lui parler de ses autres amants ; [...] elle ne lui en parla point. »

(84) Quand $\mathrm{M}^{\mathrm{me}}$ de Chartres par exemple tend un piège à sa fille en affirmant que Nemours aime la reine dauphine, $\mathrm{M}^{\mathrm{me}}$ de Clèves « en changea de visage» par jalousie (PdC, 288). Puis, apprenant indirectement par la reine dauphine que Nemours l'aime, lui, et qu'il dissimule cela par discrétion, la princesse est flattée et le fait également voir : «Si Mme la dauphine l'eût regardée avec attention, elle eût aisément remarqué que les choses qu'elles venait de dire ne lui étaient pas indifférentes,...» (PdC, 306).

(85) $\mathrm{PdC}, 319$. Elle revient à cette perspective, croyant que M. de Clèves n'a pas gardé l'aveu pour soi, en qualifiant d'erreur cette "confiance extraordinaire, ou pour mieux dire, folle que j'ai eue en vous » (PdC, 366).

(86) Pour une discussion des conditions et dangers de la «mise en discours des passions à la cour », voir W. Matzat, op. cit., pp. 241-247. 
phine le lui reproche : «Il n'y a que vous de femme au monde qui fasse confidence à son mari de toutes les choses qu'elle [fait]» $(\mathrm{PdC}, 344)$. Même M. de Clèves, qui s'était fait l'avocat de la sincérité peu avant, regrette finalement que sa femme l'ait choisi pour confident de sa passion adultère ${ }^{(87)}$. La princesse finit par affirmer avec beaucoup de confiance en elle-même : «L'aveu que je vous ai fait n'a pas été par faiblesse, et il faut plus de courage pour avouer cette vérité que pour entreprendre de la cacher » $(\mathrm{PdC}$, 352). Voilà le moment où la jeune fille se manifeste pour la première fois en tant que sujet agissant pour elle-même ${ }^{(88)}$.

Par la suite, sa lucidité psychologique augmente. Il ne revient plus à la narratrice de commenter le comportement de l'héroïne, mais à elle-même. Par exemple, lors de la découverte de l'indiscrétion de Nemours, $\mathrm{M}^{\mathrm{me}}$ de Clèves fait, après un moment de déception, preuve d'une réflexion perspicace, tout à la façon des moralistes :

Comment excuser une si grande imprudence, et qu'était devenue l'extrême discrétion de ce prince, dont elle avait été si touchée ? [...] J'ai eu tort de croire qu'il eût un homme capable de cacher ce qui flatte sa gloire. (PdC, 370)

C'est donc à partir de l'aveu, malgré ses conséquences néfastes, que la princesse commence à pénétrer le fonctionnement, puis à s'émanciper de la société de cour. La narratrice de La Princesse de Clèves part du contraste marqué entre l'habitus dominant de cette société et le comportement de l'héroïne, et décrit les étapes de sa socialisation. Cependant, elle ne s'arrête pas là. C'est le « refus » de la princesse, pendant longtemps jugé incompréhensible, qui fait suite à l'aveu dans la fonction anticipative du roman. Pendant que l'éducation sentimentale de la princesse s'accomplit, elle renonce à vivre selon les règles qu'elle vient d'apprendre et y oppose ses propres normes.

Après la mort de sa mère et de $\mathrm{M}$. de Clèves, la princesse est en effet, aux yeux de la société, libre d'épouser le duc de Nemours :

Je sais que vous êtes libre, que je le suis, et que les choses sont d'une sorte que le public n'aurait peut-être pas sujet de vous blâmer, ni moi non plus, quand nous nous engagerions ensemble pour jamais ( $\mathrm{PdC}, 408)$.

Mais, bien que tous les obstacles publics aient disparu, $\mathrm{M}^{\mathrm{me}}$ de Clèves s'oppose à une union avec Nemours, justifiant sa décision par son « devoir», mais plus encore par son « repos » $(\mathrm{PdC}, 410)$. Pour rendre compréhensible cette décision, il convient de rappeler les expériences douloureuses qu'elle vient de faire, avant tout celles de la jalousie et de l'aliénation d'elle-même. Malgré elle, elle a donné des signes de son amour à $\mathrm{Ne}$ mours ${ }^{(89)}$, lui a fait connaître sa jalousie, a abusé enfin de la confiance de son mari pour arranger une rencontre avec Nemours (la scène de la copie de la lettre de $\mathrm{M}^{\mathrm{me}}$ de Thémines). Les allusions explicites à la perte d'elle-même sont nombreuses : "L'inclination qu'elle avait pour ce prince lui donnait un trouble dont elle n'était pas maîtresse » (PdC, 309), « elle n'était plus maîtresse de ses paroles et de son visage » (PdC, 319). Enfin, elle se rend compte qu' elle est « vaincue et surmontée par une inclination qui [1']entraîne malgré [elle]» $(\mathrm{PdC}, 347)$.

$\mathrm{M}^{\mathrm{me}}$ de Clèves, résumant ses expériences, sent la honte qu'elle éprouve face à $\mathrm{M}$. de Clèves se retourner contre elle-même : ayant manqué à sa propre dignité, elle veut répa-

(87) PdC, 394. Auparavant, il avait qualifié l'aveu de « la plus grande marque de fidélité que jamais une femme ait donnée à son mari ». PdC, 352 .

(88) R. Baader, op. cit., p. 252.

(89) Bien que la princesse s'efforce de ne pas faire remarquer son inclination, « il lui échappait de certaines choses qui partaient d'un premier mouvement, qui faisaient juger à ce prince qu'il ne lui était pas indifférent. » $(\mathrm{PdC}, 313)$ 
rer ses fautes. Si, auparavant, l'aveu devait mettre fin à l'aliénation d'elle-même, c'est, après l'échec de ce premier, le refus qui devient le seul moyen par lequel la princesse peut encore espérer rétablir son moi. Par sa décision, elle se constitue en sujet autonome après avoir été successivement l'objet de sa mère, de $\mathrm{M}$. de Clèves et finalement de sa passion. Tout en renonçant à ses sentiments, elle peut les avouer, finalement même à Nemours ( $c f$. PdC, $405 s q$.). Dans la scène du refus, il n'y a plus de narratrice pour commenter et analyser la décision de l'héroïne. $\mathrm{M}^{\mathrm{me}}$ de Clèves, elle-même, présente, dans un monologue plutôt que dans dialogue avec le duc de Nemours, ses réflexions qui conduisent au refus. Ce n'est plus le moment des fausses vérités ou des signes involontaires ; c'est le temps de la conscience d'elle-même ${ }^{(90)}$.

Tandis que La Rochefoucauld se contente de constater l'échec de l'homme devant le projet d'autodétermination, $\mathrm{M}^{\mathrm{me}}$ de Lafayette montre une possibilité d'échapper à la détermination par des forces extérieures au moi : renoncer à ses passions peut mener à un acte de reconquête de soi-même ${ }^{(91)}$. Le chemin qu'elle fait faire à son héroïne est sans aucun doute épineux et douloureux (janséniste?), mais c'est le seul qui puisse l'aider à sauver ce qu'elle était en train de perdre. Ainsi, le « refus » représente la constitution du moi parfaitement conscient de ses actes et va au-delà du processus de civilisation individuelle. Le choix que fait la princesse à l'encontre de toute logique sociale - la décision de se retirer de la cour, de ses troubles et de ses bénéfices - peut dans ce sens être interprété comme un signe précurseur de l'intimité subjectivité du monde moderne à venir, même si l'ontologie de la Princesse est gouvernée ici par des motifs religieux et aristocratiques (liés au statut de la femme aussi sans doute) ${ }^{(92)}$.

La pensée d'Elias nous a permis de saisir à travers l'étude de La Princesse de Clèves la vie dans la société de cour et surtout les rapports de l'œuvre littéraire avec la logique civilisatrice. L'apparition d'un nouveau type de roman est lui-même à mettre en lien avec le processus de civilisation et les exigences de la socialisation de l'individu moderne qui se doit d'acquérir une maîtrise de soi (ses affects, son corps) intériorisée, littéralement invisible. C'est cette intériorisation de l'auto-contrainte et de l'auto-contrôle qui est au cœur du processus conflictuel de civilisation de l'hérö̈ne. La présente étude met enfin en évidence la complémentarité de l'histoire littéraire et de la sociologie historique, disciplines dont la coopération interprétative est prometteuse. Si les sciences humaines empruntaient plus souvent ces voies elles se rapprocheraient en tout cas du projet intellectuel d'Elias qui - sans relâche - a travaillé à la fécondation heuristique des paradigmes interdisciplinaires.

(90) R. Baader, op. cit., p. 262. C'est dans le même esprit que N. Grande, op. cit., pp. 100-110, réfutant $\mathrm{d}$ 'abord les hypothèses courantes que c'est par peur ou par orgueil que l'héroïne renonce au duc de Nemours, qualifie le refus d'«acte raisonnable » et cartésien (p. 105) et 1'explique par une «volonté de puissance du sujet féminin aux dépens de l'objet masculin » (p. 108).

(91) M. G. Paulson, Facets of a Princess. Multiple Readings of Madame de La Fayette's 'La Princesse de Clèves', 1998, New York, Peter Lang Publishing, pp. 1-11, qualifie le refus de « assertion of the feminine self ».

(92) Cette affirmation aristocratique d'elle-même repose sur les valeurs de «sincérité», du « repos » et du « devoir» (R. Baader, op. cit., pp. 265 et $s q$.). Ni l'aveu, ni le refus ne devraient donc être mis en relation avec la vertu bourgeoise du XVIII siècle, comme le fait R. Galle, op. cit., pp. 13-54. 


\section{Collection EthnocritiqueS - Anthropologie de la littérature et des arts}

\section{Marier les destins}

\section{Une ethnocritique des Misérables}

Cette étude ethnocritique des Misérables propose une relecture de l'œuvre majeure de Victor Hugo en articulant une poétique des textes littéraires et une anthropologie du symbolique. Elle envisage le roman à travers le prisme de son personnage principal, Jean Valjean, dont la représentation complexe emprunte ses caractéristiques à des univers culturels hétérogènes. Landen

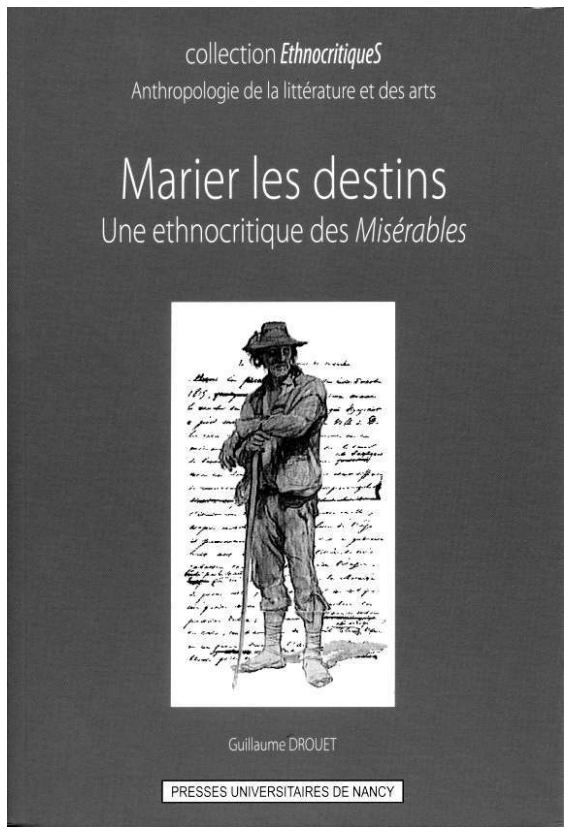

forçat suit un parcours qui le conduit à assumer les fonctions de croquemitaine, d'armièr (messager des âmes), de marieur et de pasteur. L'ensemble de ces figures, omniprésentes dans la société du XIX ${ }^{e}$ siècle et dans le texte hugolien, se combinent pour créer un personnage littéraire unique. Jean Valjean apparaît en effet comme un véritable passeur qui médiatise les relations entre adultes et enfants, hommes et femmes, vivants et morts, entre le sauvage et le domestique.

L'étude des différentes figures du héros des Misérables permet de lire son destin comme une tentative singulière d'appropriation de certaines formes de la culture du peuple (récits de croyance, superstitions, légendes et contes de tradition orale, rites et coutumes). Mais l'analyse du système des personnages (Fantine, Cosette, Marius, Gillenormand) met surtout en évidence la polyphonie culturelle de l'œuvre (le verbe hugolien synthétise et syncrétise à sa façon les tensions et interactions entre culture populaire rurale et culture bourgeoise urbaine, entre culture universelle et mythique, culture locale et historique, etc.). Jean Valjean, en mariant les destins et les cultures, propose une solution originale au problème central des Misérables : décrire et raconter l'avènement du peuple.

Docteur en langue et littérature françaises, Guillaume Drouet est enseignant dans le secondaire. Ses travaux de recherche privilégient une approche ethnocritique des littératures $d u X I X^{e}$ et $d u X X^{e}$ siècle.

Publié avec le soutien financier de la Maison des Sciences de l'Homme-Lorraine et de l'école doctorale PIEMES,

Université Paul Verlaine-Metz.

Presses universitaires de Nancy, 2011

ISBN : 978-28143-0053-8, PRIX : 20 euros 